ICRES 2019: International Conference on Robot

Ethics and Standards, London, UK, 29-30 July 2019.

https://doi.org/10.13180/icres.2019.29-30.07.010

\title{
UNDERSTANDING HOW PROXIMITY TO A ROBOT AFFECTS PERCEIVED WORKLOAD
}

\author{
TEEGAN L. JOHNSON, SARAH R. FLETCHER, AND ROLAND KUTKA
}

Industrial Psychology and Human Factors Group, School of Aerospace, Transport, and Manufacturing, Cranfield University, United Kingdom

\begin{abstract}
Human-robot collaboration is fast becoming a preferable alternative to traditional manual assembly work in manufacturing. Large industrial robots and small force-limited 'co-bots' can now be deployed with sophisticated safety systems to enable human skills and robot skills to be employed most effectively. However, an understanding of the affect this introduction may have on cognitive workload is required prior to their full installation into industry. This paper describes an investigation which explored the effect of working in proximity to a robot on people's workload. The NASA- Task Load Index (NASA-TLX) was used to assess subjective workload for participants after completing trials. Results showed centralised workload levels which were higher for the assembly activity requiring greater fine motor control.
\end{abstract}

\section{Introduction}

Within manufacturing, the expertise of operators is required for intricate and skilled craft based tasks. Their skill is valued and essential to ensuring products are produced to high standards. However, the nature of manufacturing work can place operators at risk of physical and/or psychological stress, as seen in reports release from the Health and Safety Executive [1]. Physical harm can result from lifting or carrying heavy items [2], caring out tasks in awkward postures [3] and completing repetitive tasks [4]. A solution to this would be to fully automate a task and remove operators completely from the risk of physical harm. However, this is not possible as automation and robot capabilities cannot yet replicate many essential skills completed by humans [5, 6]. Particularly craft-based tasks seen throughout manufacturing which often require fine motor control, flexibility, and the ability to adapt to new or unforeseen circumstances [7].

An increasingly proposed solution is to introduce collaborative robotic systems where the human operator and the robot work together or alongside one another. The robot would complete aspects of a task that may harm the operator or non-value added activities (retrieving and staging tools, holding parts), while the worker completes more skilled tasks [8,9]. Research has found positive 
outcomes for Human-robot collaboration (HRC) systems: Unhelkar and colleagues [9] found improved production efficiency with the introduction of a mobile robot assistant that delivered tools and materials to operators. Tan et al. [8] developed a multimodal information support system to support a HRC task. They found that the new system produced higher productivity levels with fewer assembly errors compared to conventional manual assembly. However, the interactions and proximity to the robot led to negative impacts on subjective comfort and cognitive workload. These studies demonstrate that performance benefits can be achieved by shop floor HRC. However, psychological impacts on operators have to be assessed and addressed prior to successful implementation.

\subsection{The Importance of Psychological Factors: Workload}

With the introduction of new technology, it is important to understand the costs that the technology will have on those working with it. Particularly in the transitional stage where the technology is being developed and introduced into the working environment. There are well documented instances of technology acceptance being hindered because operators have not been considered prior to installation $[5,10]$. With the introduction of automation, it was expected that high workload levels for operators would decrease. However, the opposite was found, with workload levels increasing for operators [11].

If robots are to be introduced partially to reduce the physical cost of manufacturing for operators, then the psychological cost needs to be understood and minimized where feasible. One area requiring focus is that of workload. Workload has to do the cognitive processes and relationships between task demands, the psychological resources and effort available to meet these task demands [12]. Although there are multiple processing resources, each has a limited capacity. Exposure and compensation for high levels of workload, compounded by stress resulting from organisational pressure, the introduction of new technology, or high additional attention demand, can have short term (performance deterioration [13] and longer term chronic effects on individuals $[13,14]$.

Arai and colleagues [15] found strain for human operators who did not have previous knowledge of the robots movement. This strain decreased when they were notified of motion beforehand. Interactions and proximity to robots has additionally led to negative impacts on subjective comfort and cognitive workload [8]. Together, studies such as these demonstrate that mental strain is seen with HRC will need to be fully understood to ensure effective human-robot interactions and seamless collaboration. 
Once the effect of HRC on workload has been understood, Organisations can be advised on best practice implementation methods. Therefore, this study aims to identify the effect of working in proximity to a medium sized industrial robot on workload.

\section{Method}

\subsection{Ethics}

This research was approved by the Cranfield University Research Ethics Committee, and conducted in accordance with the Cranfield Research Integrity Policy, the British Psychological Society's Code of Human Research Ethics, and the General Data Protection Regulation 2018.

\subsection{Participants}

12 participants, 8 male and 4 female aged between 21 to 46 years (mean: 33.1, SD: 8.8), participated in the study and were recruited from the staff and students from Cranfield University using random snowball sampling.

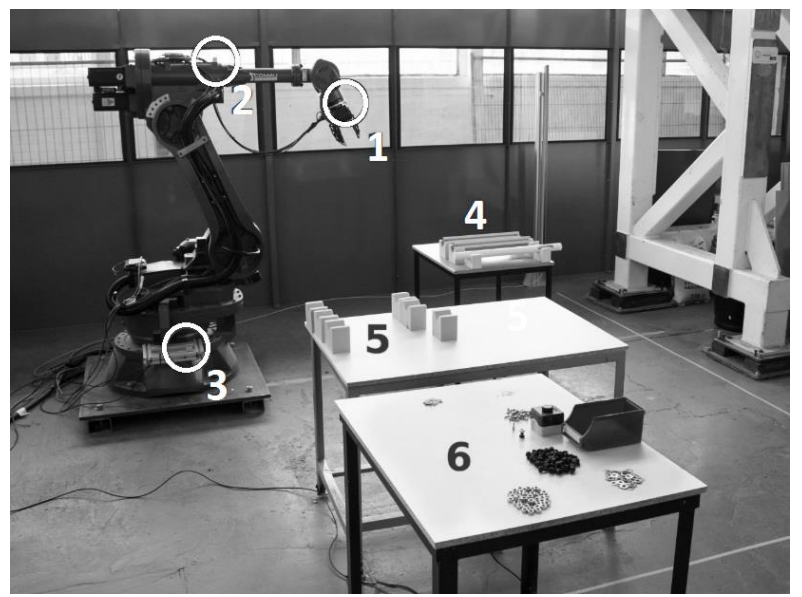

Figure 1. Experimental Trial Set-up (Numbers 1-3: Placement of LED Lights, Number 4: Pipe Start Point, Number 5: Pipe End Point, Number 6: Assembly Workbench)

\subsection{Design}

A within-subjects experimental design was used to investigate the effect of robot supervision (independent variables) on workload (dependent variable). Robot supervision was manipulated with the use of a light system and forced attention. 
3 light-emitting diodes (LED) (Q-SERIES, APEM), were placed on 3 segments of an NM45 Comau robot (Comau SpA): 1 at the top of the robot end effector, 1 on the shoulder, and one on the base (Figure 1: 1-3). To force the focus of attention participants were directed to focus their attention on the robot, the manual task, or they could choose where to focus their attention. The two conditions were counterbalanced to avoid a learning effects or association of light with the robot position [16]. All participants completed two assembly tasks (one required greater fine motor control and the second less fine motor control) (Figure 2) and reacted to the light sources by pressing a button placed on the table. Workload was measured after each trial using a NASA- TLX questionnaire [17].

\subsection{Apparatus, Laboratory Set up, and Materials}

Figure 1 shows the laboratory set up. A Comau NM 45 robot arm was used to complete a simple pick up and place task using 3 pipes. The pipes were moved from a table directly in front of the robot (Figure 1:4) to a table to the side of it and next to the participant (Figure 1: 5).

As can be seen in Figure 1 the workbench that the participants completed the assembly on was placed in front of the robot cell (Figure 1: 6). A container for completed assemblies and a button to register and turn off the lights were placed on the table. The button and lights were connected through an Allen-Bradley 1732E ArmorBlock EtherNet/IP I/O Block (Rockwell Automation, Inc) to a SoftPLC program (SoftPLC Corporation) to record the reaction times. The nuts, bolts, and washers were placed in separate piles on the table to allow for easy acquisition.

Figure A presents the two assemblies which included sets of nuts, bolts, and washers: a larger set (Task A), and a smaller set (Task B). The smaller set required greater dexterity, fine motor control, and precision, therefore it is expected greater levels of workload would be required.

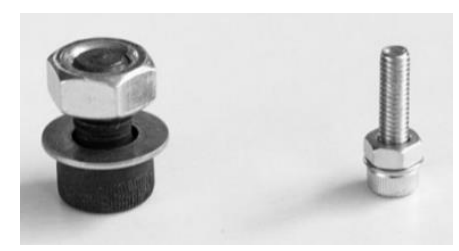

Figure 2. Task A and Task B

The NASA- Task Load Index [17] was administered to assess workload for each trial. The NASA-TLX provides an overall workload measure that has been found to have good inter-rater reliability and sensitivity to variabilities resulting 
from the weighting scheme [18]. Each time the participants completed the NASA-TLX they were provided with the rating scale definitions and completed the weightings before rating their subjective workload.

\subsection{Procedure}

Prior to participating in the experiment participants were briefed regarding their right to withdraw, confidentially and anonymity. Their consent was obtained along with their demographic information on a consent form. Participants were given a brief overview of the experiment and what would be expected of them before they were led into the laboratory area.

The participants were asked to stand side on to the robot (robot on the left side of the participant), and informed of the robots safety features. A light curtain was used to stop the robot if the curtain was broken by intrusion. The participant were asked to let the researcher know if they felt uncomfortable.

Instructions were provided on how to complete each assembly task and the final state, as seen in Figure 2. Participants were then asked to complete a pre-task assembly; this involved the completion of five assemblies for each task: A and B. Participants were then familiarised with the monitoring task, this involved each of the LEDs on the robot lighting up turning them off by pressing the button attached to the table. To avoid an association of the occurrence of a light signal with a robot state, the lights always turned on when the robot was moving.

The participants then completed six trials. At the beginning of each trial, the participant was told which Task (A or B) they should perform and where they should focus their attention for the task: on the robot, the manual assembly or their choice. Table 2 provides the details of the conditions for each task. The participants were then asked to complete as many assemblies as possible until they were told to stop. Each task was 90 seconds long. During the task each LED would turn on once. After each task the participant was asked to complete the NASA- TLX [17].

Table 2. Conditions Completed In Each Trial

\begin{tabular}{rrl}
\hline Trial & Task & Focus \\
\hline 1 & A & Manual \\
2 & A & Visual \\
3 & A & Participant Choice \\
4 & B & Manual \\
5 & B & Visual \\
6 & B & Participant Choice \\
\hline
\end{tabular}




\section{Results}

This study looked to identify the effect of working in proximity to a robot on workload, to achieve this subjective responses to the NASA-TLX were recorded. In order to produce the overall scores presented in Table 3, the mean weighted overall scores for each condition were calculated. Descriptive statistics are presented because the sample size precluded the results from statistical analysis.

All workload scores fall centrally showing a good level of workload. The lowest mean overall workload score was found for Trial 3. This involved the easier task and the greatest autonomy of focus. The highest mean overall workload score was found for Trial 6, where participants completed the more complex Task $\mathrm{B}$ which required greater dexterity, within this Trial participants were able to choose where they placed their attention.

Table 3. Means and Standard Deviations of Overall Workload for Each Trial

\begin{tabular}{rrrrrrc}
\hline & Trial 1 & \multicolumn{1}{c}{ Trial 2 } & \multicolumn{1}{c}{ Trial 3 } & Trial 4 & Trial 5 & Trial 6 \\
\hline Mean & 58.79 & 62.72 & 54.69 & 64.23 & 60.95 & 66.05 \\
$(\mathrm{SD})$ & $(11.2)$ & $(12.04)$ & $(17.5)$ & $(11.6)$ & $(11.85)$ & $(11.13)$ \\
\hline
\end{tabular}

When looking at the differences in mean scores between the Trials, it can be seen that two of the three Task A mean results were lower than their comparative Task B conditions: Trial 1 and 3 compared to Trial 4 and 6. Whereas Trial 5 (Task B, Visual Priority) had a lower overall workload score, compared to Trial 2 (Task A, Visual Priority). This is unexpected because Task B required greater dexterity and therefore it was expected that greater workload scores would be seen.

\section{Discussion and Conclusions}

The mean Overall NASA-TLX scores showed that two of the Task A Trials (1 and 3) had comparatively lower scores than their Task B counterparts (Trial 4 and 6). Therefore, indicating that Task B was subjectively more difficult to complete that Task A. This suggests that for the manual priority and the attention optional conditions, Task B required more resources to complete the trial. This was expected due to the greater dexterity and fine motor control required for Task B. However, this was not the case for the two Visual Priority Conditions (2 and 5), where the lower complexity task (Trial 2) had higher Overall scores than the high complexity task (5). A reason for this difference in the Visual priority conditions may be due to the finish positions for the nuts in each condition. Task B required the participants to run the nut to the end of the bolt, which could be complete via 
tactile feedback and consequently did not require visual attention. Whereas, the finished position for Task A required the top of the nut to be level with the top of the bolt. Therefore, this may have required visual confirmation that the nut was in the correct position, and would have removed focus from the robot and the LED lights, thereby, increasing the workload scores.

The trials within this experiment were intentionally simplified compared to those that may be observed within a manufacturing environment. Within manufacturing operators may be expected to not only press a button when they see a light signal but also make a decision and react according to the type of signal presented. Additional factors such as Organisational demands and environmental factors that may increase workload were controlled for within this study. Therefore, although the workload levels were centralized in this study it is likely that they would be higher for a more complex task that includes collaboration with a robot in a manufacturing environment. Thus, this study provides support for the research that has found that working with robots can have negative impacts on subjective cognitive workload [8], and highlights the importance of understanding this affect. Further work is required to identify the effect of more complex tasks and how working collaboratively affects workload, particularly with larger payload robots.

Future work will need conduct this experiment using a larger sample size to enable statistical analysis and identify the reliability and validity of these results. Additionally, the assessment of human robot collaboration within manufacturing and industry should be conducted to understand the other affects that interaction can have on an operator, including potential advantages. This may include looking at whether HRC can reduce the physical cost to operators and decrease non-value added activities $[8,9]$. Can human robot collaborative systems be introduced to reduce the length of time operators spend working in confined conditions because the robot can work as an additional arm to complete tasks or to provide tools in time? Or reduce the length of time participants spend working in awkward postures that with time can contribute to musculoskeletal disorders?

This study is a first step in understanding the effect of working in proximity to a robot. The intention for this is to advise organisations how best to introduce collaborative robots on to a shop floor, as well as the affect the robot will have on the operator, and the types of adjustments that will need to be made. Therefore, avoiding a repeat of previous mistakes seen with the introduction of automation [11]. 


\section{Acknowledgments.}

The Researchers would like to thank John Thrower for his support during this study.

\section{References}

1. Health and Safety Executive (2015). http://www.hse.gov.uk/statistics/industry/manufacturing/manufacturing.pdf. Accessed 09 January 2017

2. Dempsey, P.G.: A Critical Review of Biomechanical, Epidemiological, physiological and psychophysical criteria for designing manual materials handling tasks. Ergonomics. 41(1), 73--88 (1998)

3. Keyserling, W.M., Wiggermann, N., Werner, R. A., Gell, N.: Inter-Worker Variability in Lower Body Postures During Assembly Line Work: Implications for Exposure Assessment. Journal of Occupational and Environmental Hygiene. 7(5), 261--271. (2010).

4. Bao, S., Spielholz, P., Howard N., Silverstein B.: Quantifying Repetitive Hand Activity For Epidemiological Research On Musculoskeletal Disorders - Part I: Individual Exposure Assessment. Ergonomics. 49(4), 361--380 (2006).

5. Mital, A., Pennathur, A.: Advanced Technologies And Humans In Manufacturing Workplaces: An Interdependent Relationship. International Journal of Industrial Ergonomics, 33(4), 295--313, (2004).

6. Johnson, T. L., Fletcher, S. R., Baker, W., \& Charles, R. L. (2019). How and why we need to capture tacit knowledge in manufacturing: Case studies of visual inspection. Applied ergonomics, 74, 1-9.

7. Ding, Z., Hon, B.: Constraints analysis and evaluation of manual assembly. CIRP Annals-Manufacturing Technology. 62(1), 1--4 (2013).

8. Tan, J.T., Duan, F., Zhang, Y., Watanabe, K., Kato, R., Arai, T.: Human-Robot Collaboration In Cellular Manufacturing: Design And Development. IEEE/RSJ International Conference on Intelligent Robots and Systems, pp.29--34. IEEE Press, New York (2009).

9. Unhelkar, V.V., Siu, H.C., Shah. J.A.: Comparative Performance Of Human And Mobile Robotic Assistants In Collaborative Fetch-And-Deliver Tasks. In HRI '14, pp. 82--89. ACM: New York (2014)

10. Chung C.A.: Human Issues Influencing the Successful Implementation of Advanced Manufacturing Technology. Journal of Engineering and Technology Management. 13(3), 283--299. (1996).

11. Parasuraman, R., Riley, V.: Humans and Automation: Use, Misuse, Disuse, Abuse. Human Factors. 39(2), 230--253. (1997)

12. Tattersall, A.J.: Practical guidelines for workload assessment. Human Factors in Certification 1:223 (2000)

13. Hancock, P.A.: The Effect of Performance Failure and Task Demand On The Perception Of Mental Workload. Applied Ergonomics. 20(3), 197-205 (1989)

14. Hockey, R.: Compensatory Control In The Regulation Of Human Performance Under Stress And High Workload: A Cognitive-Energetical Framework. Biological Psychology. 45(1-3), 73--93 (1997) 
15. Arai, T., Kato, R., Fujita, M.: Assessment of Operator Stress Induced By Robot Collaboration. CIRP Annals - Manufacturing Technology, 59(1), 5-8 (2010).

16. Keppel, G., Saufley, W. H., Tokunaga, H.: Introduction to Design and Analysis A students Handbook. Freeman, New York (1992).

17. Hart, S. G., Staveland, L.E.: Development of NASA-TLX (Task Load Index): Results of Empirical and Theoretical Research. Advances in Psychology, 52, 139-183 (1988).

18. Hart, S.G.: NASA- Task Load Index (NASA-TLX); 20 Years Later. In Proceedings of the Human Factors and Ergonomics Society Annual Meeting. 50(9), pp.904-908 (2006). 